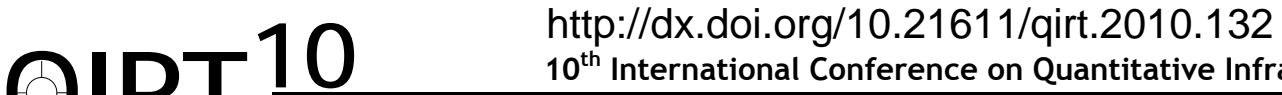 \\ $10^{\text {th }}$ International Conference on Quantitative InfraRed Thermography \\ July 27-30, 2010, Québec (Canada)
}

\section{Frequency analysis of convective cooling for the multi heat-source electronic systems - modeling and IR measurements}

\author{
by T. Świątczak*, B. Więcek*, G. De Mey** \\ * Institute of Electronics, Technical University of Łódź, Poland, tomasz.swiatczak@p.lodz.pl, wiecek@p.lodz.pl \\ ** Department of Electronics and Information Systems, Ghent University, Belgium, demey@elis.ugent.be
}

\begin{abstract}
In this article the authors focus on the research of the heat dissipation problem in electronic devices. For this purpose, a series of the numerical simulations were performed, a RC model of the electronic system with two heat sources was the object of the research. To confirm the results of the simulations, thermographic measurements were carried out. During the simulations, electronic system cooling conditions were changed as a result of varying model parameters. In the laboratory, cooling conditions of electronic system were dependent on the airflow speed around it. What is more, both simulations and measurements were performed for different positions and distances between heat sources. The authors estimated cooling conditions of the electronic system with two heat sources, with a particular interest set to the position and the distance between the heat sources. In this work the authors demonstrate, that a distribution of the heat sources in the investigated electronic system improves cooling conditions.
\end{abstract}

\section{Introduction}

This work describes thermal issues in electronic devices. This topic is of great importance, because the power density increases significantly in newer, mostly microelectronic devices when new technologies are put to production. Ubiquitous miniaturization demands from producers to use more and more efficient heat dissipation systems. What is more, increasing complexity of electronic devices and the amount of data processed by it results in higher power consumption and heat generation. Hence it is necessary to provide efficient ways of cooling electronic devices. Problems related to excessive heat dissipation in electronics are one of the major reasons hampering the development of new technologies for production of smaller generations of electronic devices, such as processors. Besides of high power densities in devices, an equally important problem is the way of its dissipation to ambience, often from the case shared with other devices. Despite of searching alternative methods of cooling the devices, still the most commonly used approach is convective cooling, often with forced air circulation [1, 2].

The authors focused on cooling conditions in multi heat-source electronic systems. The purpose of the investigation was to evaluate cooling conditions of the chosen electronic system with two heat sources, with particular interest set to the position and distance between the heat sources as well as the airflow speed around the device.

\section{Object of investigation and thermal modelling}

For the research authors have chosen a square ceramic aluminum oxide $\left(\mathrm{Al}_{2} \mathrm{O}_{3}\right)$ substrate with $50 \mathrm{~mm}$ side length and $0.8 \mathrm{~mm}$ thickness as shown in fig.1. On the top surface small square shaped resistors were deposited serving asheat sources. During the research in laboratory, the position of one of the heat sources was changed according to the arrow in fig.1, so cooling conditions were changing as a result of changing the distance between heat sources.

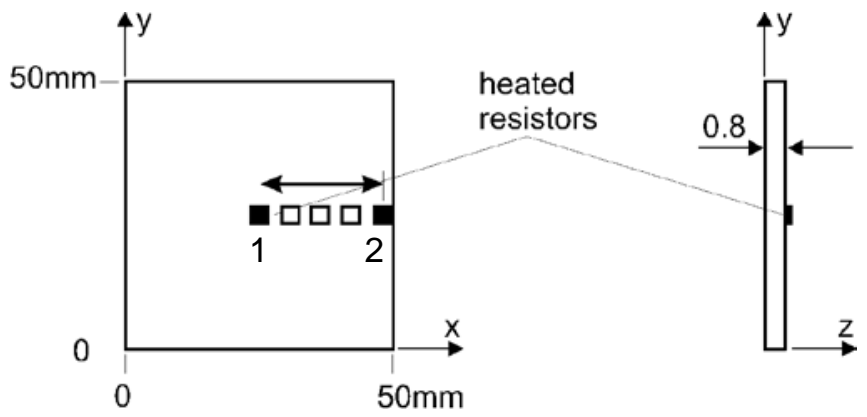

Fig.1. Aluminum oxide board with heat sources 
In the thermal model of aluminum oxide board with heat sources, thermal parameters were described with electrical ones as follows: Rth[K/W] => R[S]; T[K] => U[V]; $P[W]=>I[A] ; T a=>G N D$ (ground).

In the model the substrate was divided into small cells, which consist of the resistors and capacitors. The surface of this model had 80 by 80 cells and the model had three layers. Single connection between nodes was an equivalent of the $0,625 \mathrm{~mm}$ distance of the aluminum oxide board ( $50 \mathrm{~mm}$ divided by 80 nodes). Heat sources were modeled using 4 by 4 cells squares with current excitation, what was equivalent of the $2.5 \times 2.5 \mathrm{~mm}$ screen printed resistors. Each cell of the model had capacity, resistance to ambient and resistance to neighbour cells what is shown in fig. 2 .

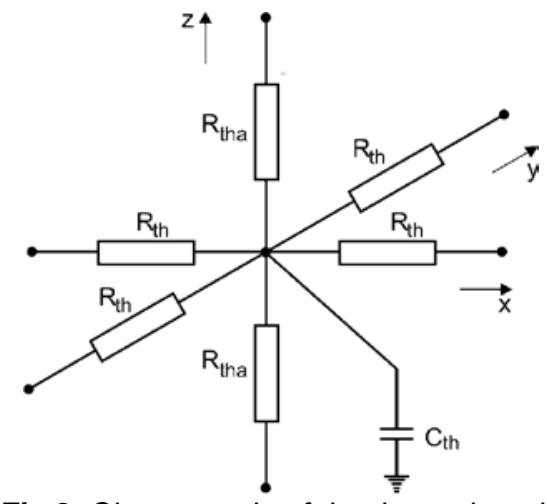

Fig.2. Chosen node of the thermal model

The thermal resistance $R_{\text {tha }}$ (board - ambient) for every cell is variable and depends on convection coefficient $h$ and surface area that dissipates heat. In addition, $R_{\text {tha }}$ does not depend on material thermal conductivity $\lambda$. The thermal resistance between particular cells of the whole investigated substrate depends on the board thickness $t$, cells dimensions and thermal conductivity coefficient. Thermal capacitance depends on board dimensions (bulk) and aluminum oxide parameters (specific heat $c_{w}$ and density $\rho$ ) [3].

\section{Simulations}

The netlist file containing the data of the model was created and the AC analysis in Spice software was performed. In this model the convection coefficient value depends on resistance between the surface of the model and ambient. This resistance was calculated analytically and next, during simulations the resistance value was changed. The dependence between convection coefficient and airflow speed was calculated too. For natural and forced convection results of the Spice simulation were obtained [3]. Calculated values of the resistance for particular convection coefficients and their equivalents of airflow speed are shown in table 1.

Table 1. Convection coefficients in simulations and airflow speed in measurements

\begin{tabular}{|c|c|c|}
\hline $\begin{array}{c}\text { Convection coefficient } \\
h\left[\mathrm{~W} / \mathrm{m}^{2} \mathrm{~K}\right]\end{array}$ & $\begin{array}{c}\text { Resistance in the model } \\
R_{\text {tha }}[\mathrm{k} \Omega]\end{array}$ & $\begin{array}{c}\text { Airflow speed in measurements } \\
v[\mathrm{~m} / \mathrm{s}]\end{array}$ \\
\hline 8,53 & 150,1 & 0 (natural convection) \\
\hline 17,66 & 72,48 & 1 \\
\hline 24,98 & 51,24 & 2 \\
\hline 33,04 & 38,74 & 3,5 \\
\hline 39,49 & 32,41 & 5 \\
\hline
\end{tabular}

What is more positions of the heat sources on the board were changed during simulations according to the arrow in fig.1. In each simulation only two heat sources were active. One of the heat sources was set in the middle of the surface and second one was moving in border direction. For different distance values between the heat sources simulations were performed (10 nodes, 20 nodes, 30 nodes and 38 nodes were chosen as a distance between heat sources). The 40 th node was the border because the model consists of 80 nodes in each direction. Results in the frequency domain were obtained for all possible cases: different convection coefficients and distances between heat sources.

Magnitude and phase of the thermal response are the results of the frequency analysis, as a response to power excitation. Thermal response of the investigated object obtained from AC simulations contained information about cooling conditions. In order to estimate convection cooling conditions, authors plotted the dependence between phase of thermal 
response and changeable parameters of the thermal model, both in function of the convection coefficient and the distance between heat sources. Results of the simulations are shown in fig. 3 below.

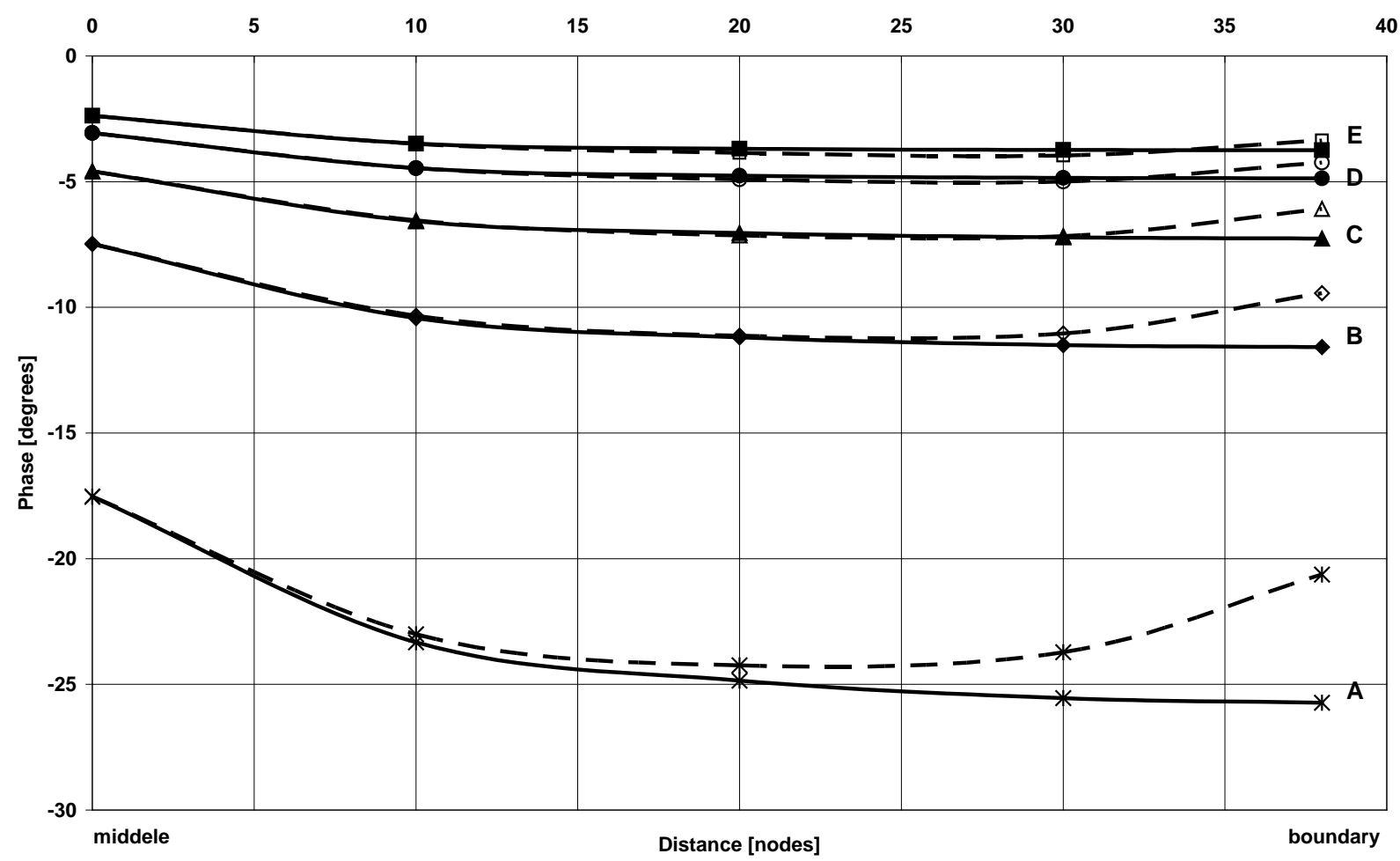

Fig.3. Results of the $A C$ simulations, plots for $1 \mathrm{mHz}$ frequency, 40 nodes is an equivalent of the $25 \mathrm{~mm}$ distance

In the figure above line plots are for the phase of the thermal response measured in the heat source in the middle of the surface. The dotted plots are for the phase observed in the heat source which position was changed during simulations. Charts "A - E "corresponds to convection coefficient $h$ values from 8,53 to $39,49 \mathrm{~W} / \mathrm{m}^{2} \mathrm{~K}$, as in the table 2 .

Table 2. Description to the fig.3

\begin{tabular}{|c|c|}
\hline $\begin{array}{c}\text { Convection coefficient } h \\
{\left[\mathrm{~W} / \mathrm{m}^{2} \mathrm{~K}\right]}\end{array}$ & Plots \\
\hline 8,53 & $\mathrm{~A}$ \\
\hline 17,66 & $\mathrm{~B}$ \\
\hline 24,98 & $\mathrm{C}$ \\
\hline 33,04 & $\mathrm{D}$ \\
\hline 39,49 & $\mathrm{E}$ \\
\hline
\end{tabular}

As it can be noted from figure above, the thermal response phase of the investigated electronic device is highly dependent on its cooling conditions. The better the cooling (convection coefficient is higher), the lower the absolute values of the thermal response phase may be observed in particular nodes. In addition, the higher is the distance between sources, the higher the absolute value of the thermal response phase can be observed, in the middle node.

In the investigated case the difference between absolute phase values measured in two heat sources increases with the distance between them. This is because of the "mirroring" effect, which causes the absolute phase value of the side heat source to increase when it reaches the proximity of the board border. The explanation of this phenomenon lies in a boundary condition, which states that one can assign a mirror reflection of the moving heat source with respect to the board border. When the heat source gets closer to the board edge, it becomes closer to its "reflection", therefore decreasing the absolute phase value. 


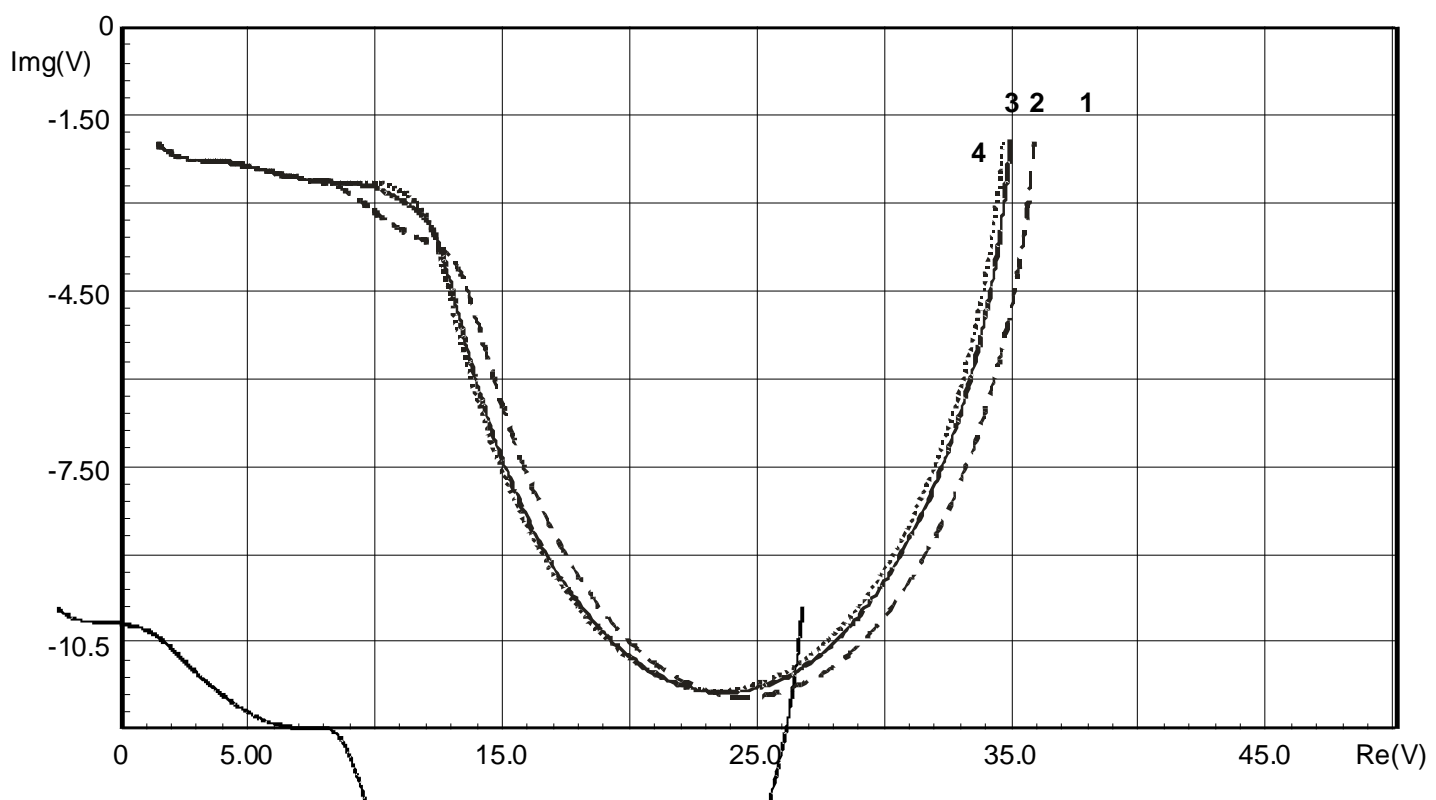

Fig. 4. Results of the AC analysis - Nyquist plot fdr the heat source in the middle of the board

In fig.4 the Nyquist plots repre sent the thermal response for different distances between heat sources on the surface for convection factor $h=8,53 \mathrm{~W} / \mathrm{m}^{2} \mathrm{~K}$ (natural convection). Pløt 1 corresponds to a distance of 10 nodes between the heat sources, plot $2-20$ nodes, plot $3-30$ nodes, plot $4-38$ ngdes (boundary of the substrate). The obtained results confirmed that the phase value of the thermal response depends on fonvection cooling conditions (convection coefficient) and distance between heat sources. The increase of the distance between the heat sources results in position translation of the Nyquist plot, to the left. This is because the cooling conditions are better when the distance between heat sources is higher.

\section{IR measurements}

The aluminum oxide substrate with resistors, which was the subject of research, was placed in a low speed wind tunnel that enabled to change the cooling conditions thanks to the possibility of adjusting the airflow speed. Series of pulse thermography measurements were carried out during the heating and cooling this system by two heat sources (resistors) simultaneously. Pulse excitation measurements were performed for each source heated with 1,5 W power. Thermal response in the time domain of this electronic system was observed with thermographic camera Jenoptik VarioCAM HiRes ${ }^{\circledR}$. Sequences of thermograms were recorded for different cooling conditions and different positions of one of the heat sources. For each arrangement of the resistors, the authors recorded a sequence of thermograms for natural convection and forced one, with airflow speeds as shown in table 1.
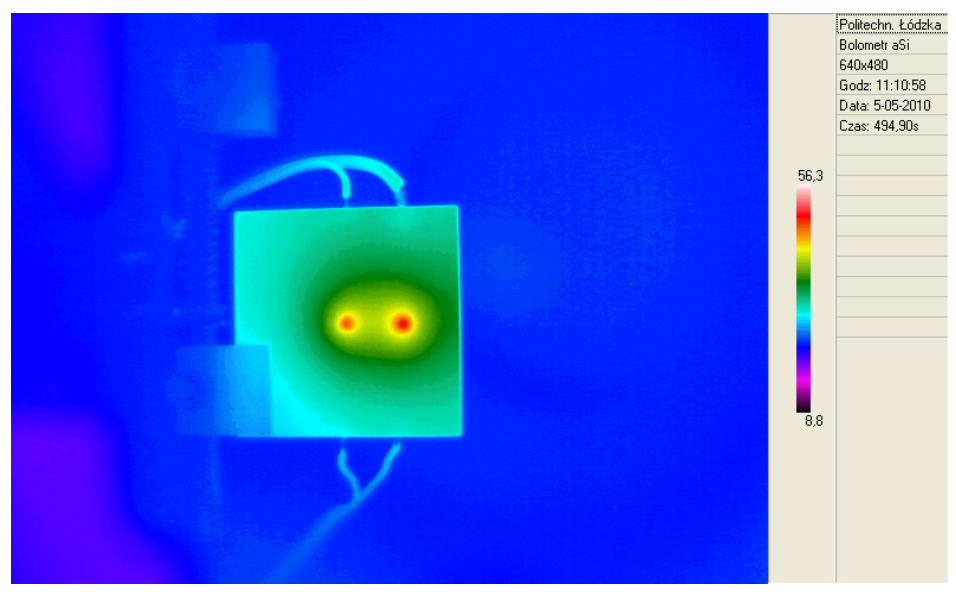

Fig.5. Exemplary frame of the thermogram sequence for natural convection. 
Using ThermalScope and Matlab software, a FFT transform was calculated for each single pixel along all thermograms from the recorded sequences. The result was a frequency response of the set-up for thermal excitation. The calculated phase and amplitude of the thermal response of the board was subjected to the detailed analysis with particular interest set to phase diagrams.

FFT enables to convert signal, from time to frequency domain, and to calculate phase of the thermal response for desired frequency. The recorded sequence of one pulse excitation lasted 1000 seconds, therefore authors had chosen the frequency of the first harmonic $1 \mathrm{mHz}$, and for this frequency evaluated the dependence between the phase of thermal response and the cooling conditions (airflow speed). However, authors focused on determination of the thermal response phase versus distance between heat sources.

In the figures below, the plots show the dependence between the phase of the thermal response and the distance between heat sources, measured for natural convection. The authors compared results of the simulation and the experimental measurements for all arrangements of investigated heat sources. In the fig.5 and fig.6, ox axis indicates the distance between heat sources and oy axis indicates the phase of the thermal response for pulse excitations.

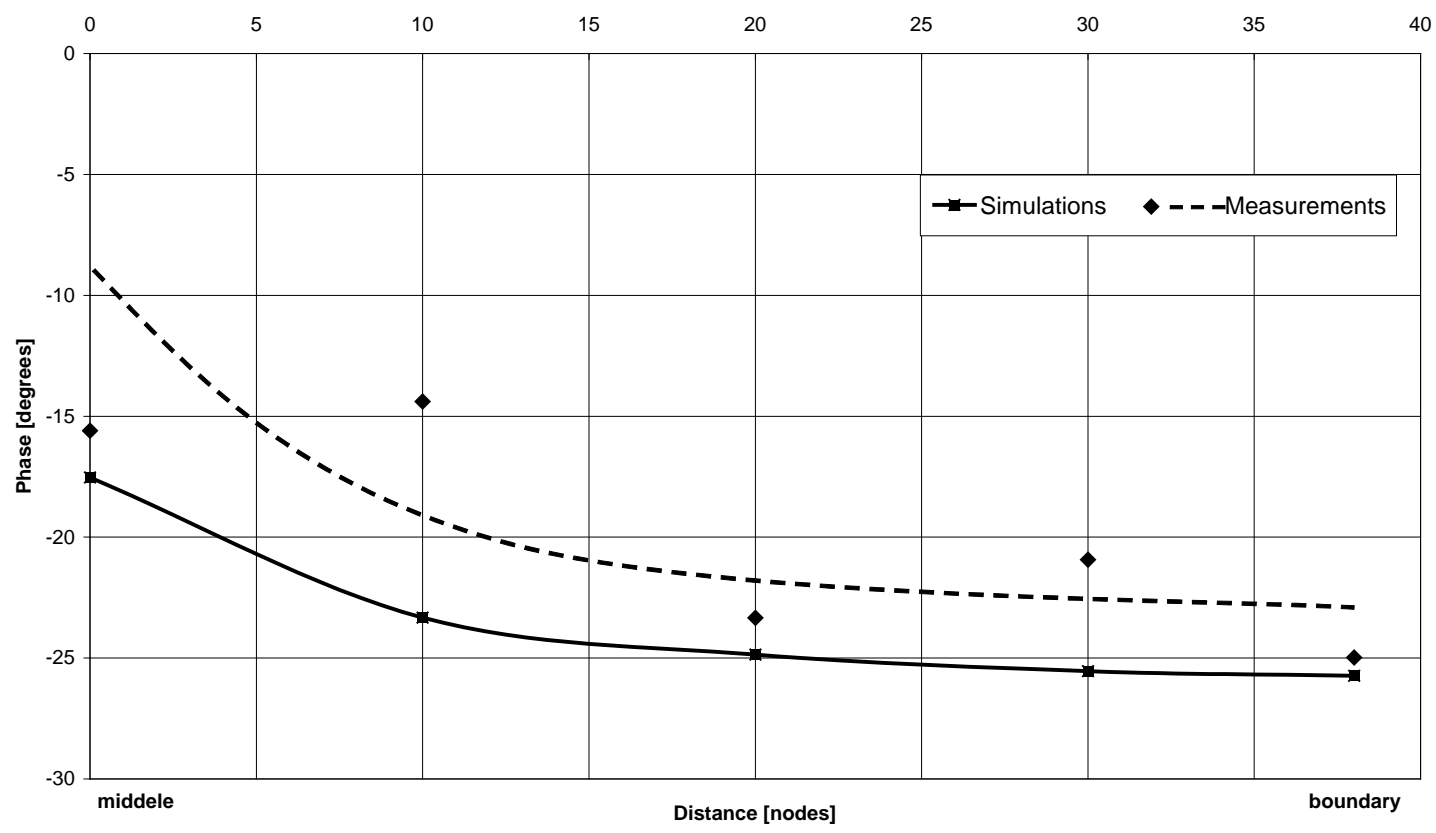

Fig.6. Phase of the thermal response versus distance between heat sources, measured in the middle heat source, for natural convection; 40 nodes is an equivalent of the $25 \mathrm{~mm}$ distance

As it can be noted from fig.5 and fig.6 (above and below), the measurements confirmed the results of the simulations, despite minor measurements errors, that arose due to the equipment uncertainty. Hence it was necessary to perform an approximation of the experiment data. 


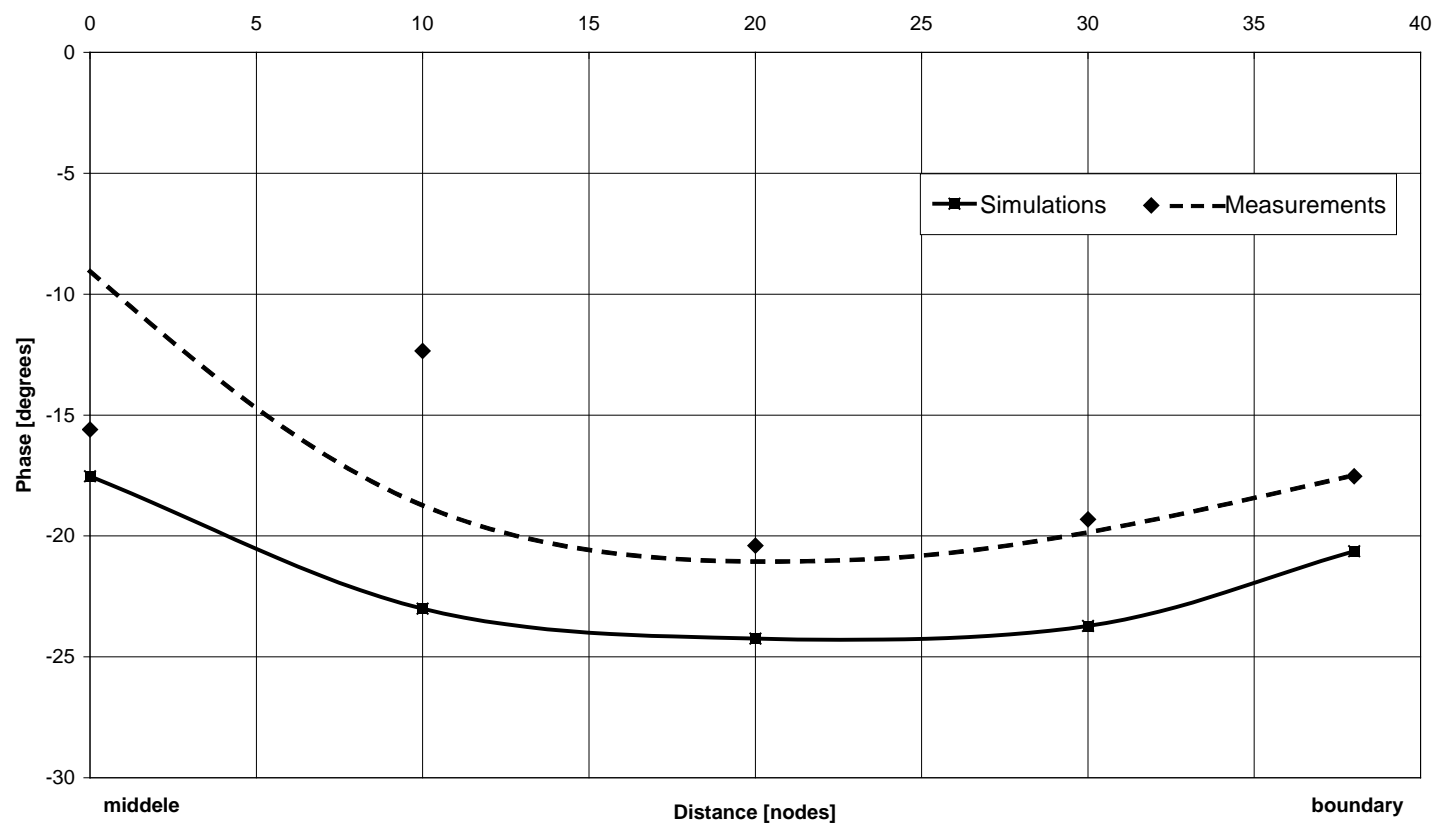

Fig.7. Phase of the thermal response versus distance between heat sources, measured in the side heat source, for natural convection; 40 nodes is an equivalent of the $25 \mathrm{~mm}$ distance

In addition, authors determined the thermal impedance of the measured surface with heat sources. For the chosen configuration of the heat sources and for natural convection, the Nyquist plot of the thermal impedance (Zth) was evaluated.

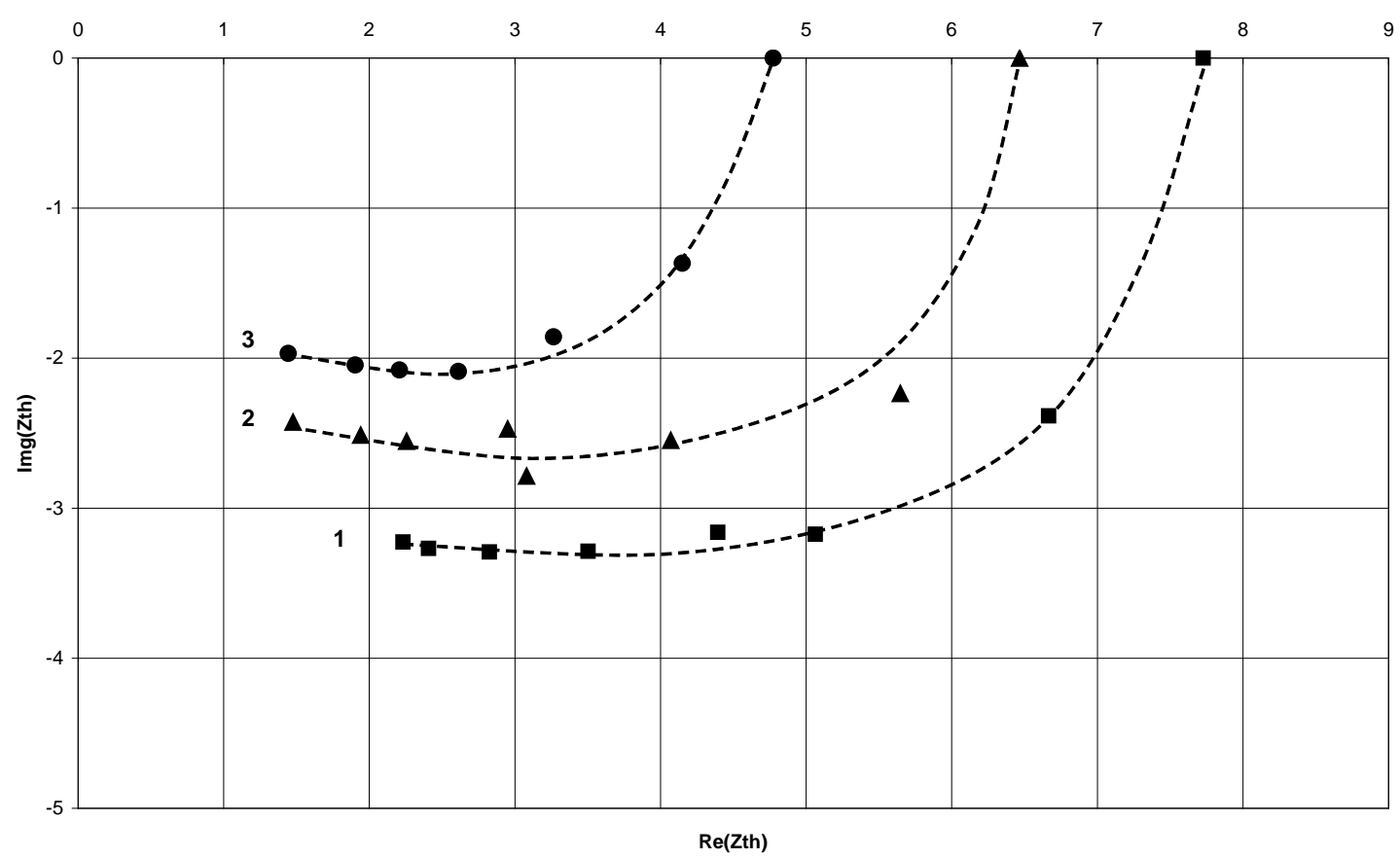

Fig.8. Nyquist plot of the thermal impedance Zth, for natural convection 
In fig.8 the Nyquist plots represents the thermal impedance Zth for different distances between heat sources (resistors) on the surface for natural convection $\left(h=8,53 \mathrm{~W} / \mathrm{m}^{2} \mathrm{~K}\right)$. Plot 1 corresponds to the distance of 20 nodes between heat sources, plot $2-30$ nodes, plot $3-38$ nodes (boundary of the substrate).

As it can be noted from the figure above, the real part of the thermal impedance $(Z$ th $)$ of the investigated object decreases with increasing distance between heat sources. Hence the higher the distance between the heat sources is, the better the cooling conditions are.

\section{Conclusions}

To sum up the results of the described investigation, the absolute phase value of the thermal response increase with the distance between the heat sources. The higher is the distance between heat sources, the better are the convective cooling conditions. The measurements confirmed that the thermal impedance is decreasing in function of the distance between the heat sources. In conclusion, heat sources should be evenly arranged on the whole surface of the electronic system to improve cooling conditions.

To draw a final conclusion, one may state that using a pulse thermography technique, which is one of nondestructive examination methods, it is possible to estimate the convective cooling conditions of the measured set-up. The recorded sequences of thermograms are to be transformed to the frequency domain using FFT, in order to be used as a base to conclude about the conditions of cooling of particular electronic device. In case of this paper, using this method, it was possible to estimate the convection cooling condition in function of the distance between two heat sources.

Positive results, that were obtained, enabled authors to conclude that proposed method may be widely applied in electronics to estimate the convective cooling conditions and the quality of thermal connections.

Tomasz Świątczak thank to Ministry of Science and Higher Education of Poland for the financial support (ref. no. 3601/B/T02/2009/36).

Tomasz Świątczak is scholarship holder of the project "Innovative didactics without limitations - integrated development of The Technical University of Łódź - management of the university, modern educational offer and increasing potential to employ also people with disabilities" co-financed by the European Union within the European Social Fund.

\section{REFERENCES}

[1] T. Świątczak, R. Olbrycht, B. Więcek "Evaluation of convection cooling conditions using Fourier and wavelet analysis in lock-in thermography", 9th Quantitative Infrared Thermography conference, QIRT2008, July 2-5, 2008, Krakow, Poland.

[2] S. Wiśniewski „Wymiana ciepła”, WNT, 2000, in Polish.

[3] T.Świątczak, B.Więcek, K.Tomalczyk "Convective cooling evaluation of electronic devices using lock-in thermography", 14 th International Conference, MIXDES 2007, June 21-23 2007, Ciechocinek, Poland.

[4] G. De Mey, J. Pilarski, M. Wójcik, M. Lasota, J. Banaszczyk, B. Vermeersch and A. Napieralski, "Influence of interface materials on the thermal impedance of electronic packages", International Communications in Heat and Mass Transfer, Volume 36, Issue 3, March 2009.

[5] G.De Mey, B.Vermeersch, J.Banaszczyk, T.Świątczak, B.Więcek, M.Janicki, A.Napieralski "Thermal impedances of thin plates", International Journal of Heat \& Mass Transfer, ScienceDirect, Elsevier; Vol. 50 Issues 21-22 October 2007.

[6] A. Gleiter, C. Spießberger, G. Busse „Phase Angle Thermography for depth resolved defect characterization”, 9th Quantitative InfraRed Thermography conference, QIRT2008, July 2-5, 2008, Krakow, Poland. 